\title{
Designing a Wearable Vibrotactile Feedforward Wrist Display for Novice Gameplay
}

\author{
Katie Seaborn and Alissa N. Antle \\ School of Interactive Arts and Technology, Simon Fraser University, \\ 250 - 13450 102nd Avenue, Surrey, BC, Canada V3T 0A3 \\ \{kseaborn, aantle\} asfu.ca
}

\begin{abstract}
As the potential of the video game medium expands, new design challenges come to light. In this paper, we present the design of a wearable vibrotactile feedforward display for novice players. The device, named "The Gauntlet Guide," is designed to provide vibrotactile guidance cues during navigation tasks in a fast-paced, visual- and audio-intensive video game. We report on a preliminary study in which we assessed the reliability, ergonomics and appeal of the display. Our findings support continued research on haptic feedforward as a viable technique for scaffolding the learning curve in modern video game environments and engaging novice players in gameplay.
\end{abstract}

Keywords: Vibrotactile, feedforward, guidance, tactile wrist display, wearable, haptics, video games, learning curve, navigation, novice players.

\section{Introduction}

As a maturing medium with a solid foundation in the entertainment industry, the video game is increasingly being embraced outside of entertainment contexts. This raises the issue of how to design video games for new purposes (e.g. learning, training) and previously overlooked audiences (e.g. children, women). In this inprogress research, we focus on three central aspects of the video game medium: its expanding audience, increasing sophistication, and multimodal nature. These foci guided us in the design of a prototype for engaging novice players who are unfamiliar with navigating modern game environments. In this paper, we present insights from our design process and report on findings from a preliminary study.

\section{Research Problem}

The learning curve necessary to engage with increasingly sophisticated game environments can make them inaccessible to novice players [1]. Novice players can expend effort on navigation, which distracts them from main gameplay tasks [2]. A potential solution that has not been adequately explored is feedforward [3]. Feedforward, contrasted with feedback, is a system response intended to guide future action; only recently has feedforward gained attention. We seek to explore its uncharted potential in the design of our prototype. 
The multimodal aspect of modern game environments lends itself to the use of haptics for feedforward. In a previous study that compared visual and haptic modalities, we found no indication of simultaneous visual and haptic stimuli hampering performance in a pattern-matching task [4]. Moreover, Forsyth and MacLean [5] conducted a study that showed increased performance with and preference for the use of predictive, overlaid haptic cues in dynamic tasks that involved differing path complexity and visibility. These findings suggest that haptic guidance could be well suited to a gaming context, where maintained engagement and agency is crucial. However, this needs to be explored empirically.

A great deal of research has been conducted around the concept of wearable tactile displays (WTD) for navigation [6]. However, only recently has the use of vibrotactile stimuli for presenting discreet secondary information gained attention. Lee and Starner [7] developed a WTD that presents two-dimensional navigation information via directional vibration cues. Their design features a 3x3 array of actuators, which allows for a rich selection of patterns. However, users need to undergo 40 minutes of training to perform at $99 \%$ accuracy. Such a design, while rich with regard to information presentation, presents issues for the problem under question in our research because our goal is to support early and consistent gameplay for novice users. In contrast, initial findings from our preliminary study indicated that our simpler design requires at most 10 minutes of training [8]. However, the implications of this are contingent upon our prototype's success in the upcoming full study.

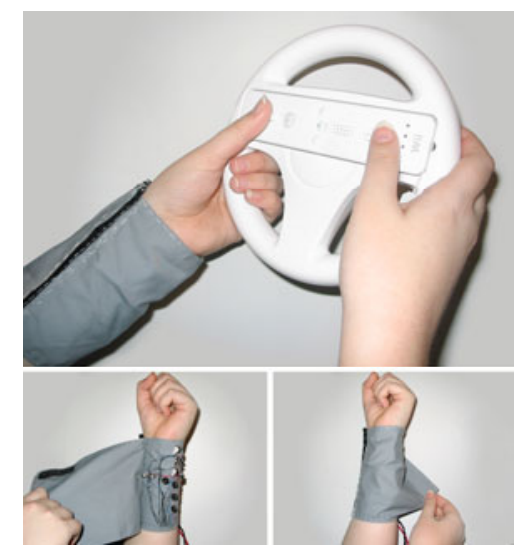

Fig. 1. The Gauntlet Guide coupled with the Wii Wheel. The bottom two photos show how the actuators are covered.

\section{The Gauntlet Guide}

Our wearable wrist display provides vibrotactile navigation cues via coin type pager motors arranged in the cardinal directions along the wrist. Players receive haptic feedforward information via the display and control their game avatar using the Wii Wheel, the controller that comes with our chosen fast-paced, multimodal video game, Mario Kart Wii (2008). As the novice player learns how to use the device, they are in 
the process of crafting a mental model of how the device works; we have coupled these elements to support this process, as suggested by the spatial contiguity principle, one of Mayer's multimedia principles for learning.

The directional patterns themselves garnered special consideration during the ideation stage due to our focus on navigation and known stimuli discrimination problems with respect to vibrotactile motion. We considered variations in spatial configuration and motion style. We implemented a novel mounting for the actuators: instead of being laid flat, the actuators are positioned perpendicular to the wrist.

Positive findings from our preliminary study support the continued exploration of this mounting in the full study [8]. Further, we discovered that sequential motioneach actuator in a given direction being triggered in sequence-was preferred. Our "attention-grabbing" play of the first stimulus opposite to the actual sequence was found to be unsuitable. This led us to use the first stimulus in the sequence instead. Finally, given observations of orientation difficulties, the prototype is now worn along the left side of the left wrist, complimenting how the Wii Wheel is held.

\subsection{Future Research: Evaluation in Context of Use}

In the next phase of this research, we will evaluate our prototype in its intended context of use. We propose a within-subjects study to investigate how augmenting navigation in a fast-paced multimodal game environment with haptic feedforward affects the user experience of novice players. We ask: Is haptically augmented feedforward an effective style of guidance for novice players navigating a fast-paced game environment that commands the attention of visual and auditory modalities?

We will employ a Wizard of $\mathrm{Oz}$ setup, involving a human operator enacting computer responses; this approach has been widely validated for HCI studies. The main task will involve playing through three levels of a racing game with each of three feedforward configurations: haptic, visual and none. The comparison of visual and haptic presentation modes draws directly from our previous work comparing light and heat [9] and visual and haptic modes [4]. Game level and feedforward configuration will be counter-balanced to account for carry-over effects.

We will measure performance (task time and errors), satisfaction (in a post-task questionnaire comprised of Likert scale questions drawn from Davis's scale for perceived ease of use with information technology [10]) and engagement (in selfreports using the Game Engagement Questionnaire [11]).

\subsection{Contributions}

Our goal is to explore the potential of haptic feedforward through a vibrotactile display for guiding novices. We propose three contributions:

1. Knowledge: To discover how augmenting navigation in a sophisticated game environment with haptic feedforward affects the user experience of novice players.

2. Demonstration: To exhibit a proof-of-concept approach through a prototype-the Gauntlet Guide-that seeks to increase the user experience potential of haptics in gaming through a new context of use.

3. Principles: To establish a set of design guidelines for haptic feedforward. 
We seek to explore the potential of haptic feedforward, an approach whose value we foresee increasing as the video game medium continues to push the boundaries of technical quality, applications and audience.

Acknowledgements. Our thanks to Dr. Bernhard E. Riecke and our lab group (Randa Aljohani, Ying Deng, Daniel Feuereissen, Lonnie Hastings, Anna Macaranas, Andrew Milne, Salvar Sigur-darson, Tess Speelpenning, Jay Vidyarthi) for their support, ideas, and feedback. We are funded by the Social Sciences and Humanities Research Council of Canada.

\section{References}

1. Becker, K.: Distinctions Between Games and Learning: A Review of Current Literature on Games in Education. In: Gaming and Cognition: Theories and Practice from the Learning Sciences, pp. 22-54. IGI Global (2010)

2. Virvou, M., Katsionis, G.: On the usability and likeability of virtual reality games for education: The case of VR-ENGAGE. Computers \& Education 50, 154-178 (2008)

3. Swan, R.H.: Feedforward as an Essential Active Principle of Engagement in Computer Games. In: Gaming and Cognition: Theories and Practice from the Learning Sciences, pp. 108-136. IGI Global (2010)

4. Seaborn, K., Riecke, B.E., Antle, A.N.: Exploring the interplay of visual and haptic modalities in a pattern-matching task. In: Proceedings of the 9th IEEE International Symposium on Haptic Audio Visual Environments and Games, pp. 61-66. IEEE, Phoenix (2010)

5. Forsyth, B.A.C., MacLean, K.E.: Predictive Haptic Guidance: Intelligent User Assistance for the Control of Dynamic Tasks. IEEE Transactions on Visualization and Computer Graphics 12, 103-113 (2006)

6. Matscheko, M., Ferscha, A., Riener, A., Lehner, M.: Tactor placement in wrist worn wearables. In: 2010 International Symposium on Wearable Computers (ISWC), pp. 1-8 (2010)

7. Lee, S.C., Starner, T.: BuzzWear: alert perception in wearable tactile displays on the wrist. In: Proceedings of the 28th International Conference on Human Factors in Computing Systems, pp. 433-442. ACM, Atlanta (2010)

8. Seaborn, K., Antle, A.N.: Gauntlet Guide: Designing a Wearable Vibrotactile Feedforward Display for Novice Gameplay. In: Proceedings of GRAND Annual Conference 2011, Vancouver, British Columbia, Canada (2011)

9. Seaborn, K., Antle, A.N.: The Tiresias Effect: Light versus Temperature in a Tangible User Interface. In: Proceedings of the 28th International Conference Extended Abstracts on Human Factors in Computing Systems, pp. 3427-3432. ACM, Atlanta (2010)

10. Davis, F.D.: Perceived usefulness, perceived ease of use, and user acceptance of information technology. MIS Quarterly 13, 319-339 (1989)

11. Brockmyer, J.H., Fox, C.M., Curtiss, K.A., McBroom, E., Burkhart, K.M., Pidruzny, J.N.: The development of the Game Engagement Questionnaire: A measure of engagement in video game-playing. Journal of Experimental Social Psychology 45, 624-634 (2009) 